\title{
Healthcare Costs and Resource Utilization Associated with the Use of Empagliflozin Versus Other Antihyperglycemic Agents Among Patients with Type 2 Diabetes Mellitus and Cardiovascular Disease: A Real-World Retrospective Cohort Analysis
}

\author{
Aditya Raju (D) P Pratik Pimple · Dana Stafkey-Mailey • \\ Eileen Farrelly $\cdot$ Sharash Shetty
}

Received: August 26, 2021 / Accepted: October 8, 2021 / Published online: November 2, 2021

(C) The Author(s) 2021

\section{ABSTRACT}

Introduction: Empagliflozin has demonstrated lower rates of cardiovascular outcomes vs. standard of care among patients with type 2 diabetes mellitus (T2DM) and cardiovascular disease (CVD). However, the impact of empagliflozin compared to other branded antihyperglycemic agents (AHAs) on total cost of care has yet to be quantified.

Methods and Results: This retrospective cohort study evaluated the impact of empagliflozin $(n=441)$ on costs and healthcare resource utilization (HCRU) vs. other branded AHAs

\footnotetext{
A. Raju $(\varangle) \cdot$ D. Stafkey-Mailey · E. Farrelly

Xcenda LLC, Carrollton, TX, USA

e-mail: aditya.raju@xcenda.com

D. Stafkey-Mailey

e-mail: dana.stafkey-mailey@xcenda.com

E. Farrelly

e-mail: eileen.farrelly@xcenda.com

P. Pimple $\cdot$ S. Shetty

Boehringer Ingelheim Pharmaceuticals Inc.,

Ridgefield, CT, USA

P. Pimple

e-mail: pratik.pimple@boehringer-ingelheim.com

S. Shetty

e-mail: sharashchandra.shetty@boehringer-

ingelheim.com
}

$(n=13,122)$ among patients with T2DM and CVD, using the IQVIA PharMetrics ${ }^{\circledR}$ Plus Claims Database (1 August 2013-31 December 2017). Date of the first prescription (index date) for empagliflozin or other branded AHAs was used to classify patients into study cohorts. All-cause costs and HCRU were computed on a per patient per month (PPPM) basis and compared across study cohorts using outcome-appropriate statistical models. Overall, the empagliflozin cohort was younger and had a lower comorbidity burden. After covariate adjustment, the total all-cause costs (mean difference - \$412 PPPM; 95\% CI - \$593, - \$214) were significantly lower for the empagliflozin cohort. These cost differences were mainly driven by lower all-cause medical costs (mean difference - \$400 PPPM; 95\% CI - \$577, - \$196). For HCRU, the mean adjusted all-cause visits in the physician office and other outpatient settings were lower with empagliflozin vs. other branded AHAs $(p<0.001)$.

Conclusions: This study demonstrated that the all-cause healthcare costs and HCRU were significantly lower for patients with T2DM and CVD who initiated empagliflozin vs. other branded AHAs. Along with the positive clinical evidence base of empagliflozin, these results can guide healthcare decision makers during therapy selection. 
Keywords: Costs; Type 2 diabetes mellitus; Cardiovascular disease; Empagliflozin; Healthcare resource utilization

\section{Key Points}

Why carry out this study?

The cardioprotective benefits of empagliflozin among patients with type 2 diabetes mellitus (T2DM) and cardiovascular disease (CVD) are well established in the literature; however, evidence of its impact on costs and healthcare resource utilization (HCRU) in this population is limited.

This study compared the all-cause costs and HCRU among patients with T2DM and CVD who initiated treatment with empagliflozin versus other branded antihyperglycemic agents (AHAs).

\section{What was learned from the study?}

Initiation of empagliflozin versus other branded AHAs among patients with T2DM and CVD was associated with lower allcause costs and resource utilization.

Results from this study highlight the economic benefits of empagliflozin; and when taken together with the clinical benefits, these data can aid healthcare stakeholders during therapeutic or policy decisions for patients with T2DM and CVD.

\section{INTRODUCTION}

\section{Background}

The clinical and economic burden of type 2 diabetes mellitus (T2DM) and its complications continues to rise, despite significant therapeutic advances in the last decade. The most prevalent complication of T2DM is cardiovascular disease (CVD), where individuals with T2DM are two to four times more likely to develop CVD than those without T2DM. Consequently, CVD is the leading cause of morbidity and mortality in patients with T2DM [1-3].

The comorbidity burden and complications associated with CVD in patients with T2DM also contribute substantially to costs of managing the disease $[4,5]$. A recent global review reported that the average annual allcause cost to treat patients with T2DM without comorbid CVD was $\$ 8310$ compared to $\$ 15,105$ for patients with T2DM and comorbid CVD [5]. This represented a median increase in burden of $112 \%$ (range 47-196\%). Furthermore, the review also estimated the costs of comorbidities to be $20 \%$ to $49 \%$ of the total costs of T2DM, with a weighted average of $42 \%$ for CVD [5]. In the USA, the cost associated with diagnosed diabetes was $\$ 327$ billion in 2017 , of which approximately $\$ 37.3$ billion was from CVD-related expenditures. Accordingly, CVD also leads to increased healthcare resource utilization (HCRU) in patients with T2DM, including increased hospital inpatient stays and emergency department (ED) visits compared to patients with T2DM without CVD [4].

While there are many available treatment options for T2DM, it may be prudent to consider drugs that have demonstrated a beneficial impact for cardiovascular (CV) outcomes in addition to glycemic control. Sodium-glucose cotransporter 2 inhibitors (SGLT2is) are a class of agents used to treat T2DM that have a unique mechanism of action, where agents in this class inhibit glucose and sodium reabsorption in the proximal tubule of the kidney, which in turn increases urinary glucose excretion and natriuresis [6]. In large-scale clinical trials of patients with T2DM and either established CVD or the presence of multiple risk factors for CVD, SGLT2is have demonstrated CV benefits [7-10]. The beneficial effects have also been documented in real-world studies [11-15].

\section{Study Rationale and Purpose}

The EMPA-REG OUTCOME trial, which was designed to determine the long-term CV safety of empagliflozin, an SGLT2i, in patients with 
T2DM and atherosclerotic CVD, demonstrated lower rates of $\mathrm{CV}$ outcomes for empagliflozin relative to standard of care. Specifically, empagliflozin showed a $38 \%$ relative risk reduction in CV death (hazard ratio [HR] 0.62; 95\% confidence interval $[\mathrm{CI}] 0.49,0.77), 35 \%$ relative risk reduction in hospitalization for heart failure (HHF) (HR 0.65; 95\% CI 0.50, 0.85), 32\% relative risk reduction in all-cause death (HR 0.68; $95 \%$ CI $0.57,0.82$ ), and $14 \%$ relative risk reduction in the composite three-point major adverse cardiovascular events (MACE) outcome (HR 0.86; 95\% CI 0.74, 0.99) [7, 16]. Real-world evidence on the effects of SGLT2is on these outcomes also aligns with the trial data; recent studies found lower rates of heart failure-related hospitalizations and/or all-cause mortality among patients who initiated treatment with an SGLT2i compared to patients initiating other glucose-lowering drugs [12, 17-20]. In addition, the 2018 American College of Cardiology (ACC) Expert Consensus Decision Pathway and 2019 American Diabetes Association Standards of Medical Care in Diabetes recommends use of either an SGLT2i (empagliflozin being the preferred agent) or a glucagon-like peptide 1 receptor agonist (GLP-1RA) in patients with T2DM and CVD [21, 22]. The evidence-based guidelines further reinforce the use of empagliflozin in patients with T2DM and CVD from a clinical perspective; however, the impact of empagliflozin compared to other branded antihyperglycemic agents (AHAs) on healthcare utilization and total cost of care is yet to be quantified.

There is a recognized gap in the literature with regards to a clear understanding of the economic impact of therapy choice. This study sought to provide healthcare decision makers with evidence to help guide therapy selection with regards to economic burden. The study evaluated the impact of empagliflozin on costs and HCRU relative to other branded AHAs, which included oral agents like dipeptidyl peptidase 4 inhibitors (DPP4is) or other SGLT2i drugs, and injectable agents such as GLP-1RAs or insulin, among patients with T2DM and established CVD.

\section{METHODS}

\section{Data Source and Study Design}

This was a retrospective cohort study that used integrated healthcare claims from the IQVIA PharMetrics ${ }^{\circledR}$ Plus Claims Database. This database contains data from over 103 different managed healthcare plans, as well as Blue Cross Blue Shield data. The payer type distribution for this data source is $80 \%$ commercial, 3\% Medicaid, $1.7 \%$ Medicare risk, and the rest are categorized as "other". This database contains deidentified administrative claims data per the US Health Insurance Portability and Accountability Act (HIPAA); hence, institutional review board approval was not required for this study.

Patients with T2DM and established CVD who newly initiated treatment with either empagliflozin or other branded AHAs belonging to any of the following four classes were studied from August 1, 2013 through December 31, 2017 (study period; see Fig. 1): DPP4i, other SGLT2i (besides empagliflozin), GLP-1RA, or insulin. For patients receiving empagliflozin during the identification period (August 1, 2014 through December 31, 2016), the index date was defined as the date of the first prescription for empagliflozin; for the remaining patients who did not receive empagliflozin and received other branded AHAs during the identification period, the index date was the date of the first prescription for other branded AHAs. The preindex period, which was defined as 12 months prior to index date, was used to identify patients with both CVD and T2DM diagnosis and to assess baseline characteristics. The post-index period was used to assess outcomes and included a variable period from the index date until the earliest of (a) discontinuation of index drug of interest (i.e., treatment gap of at least 60 days), (b) addition of a different branded AHA, (c) end of 12-month post-index period, or (d) end of continuous health plan eligibility. A minimum of a 2-month post-index period was required for study inclusion. 


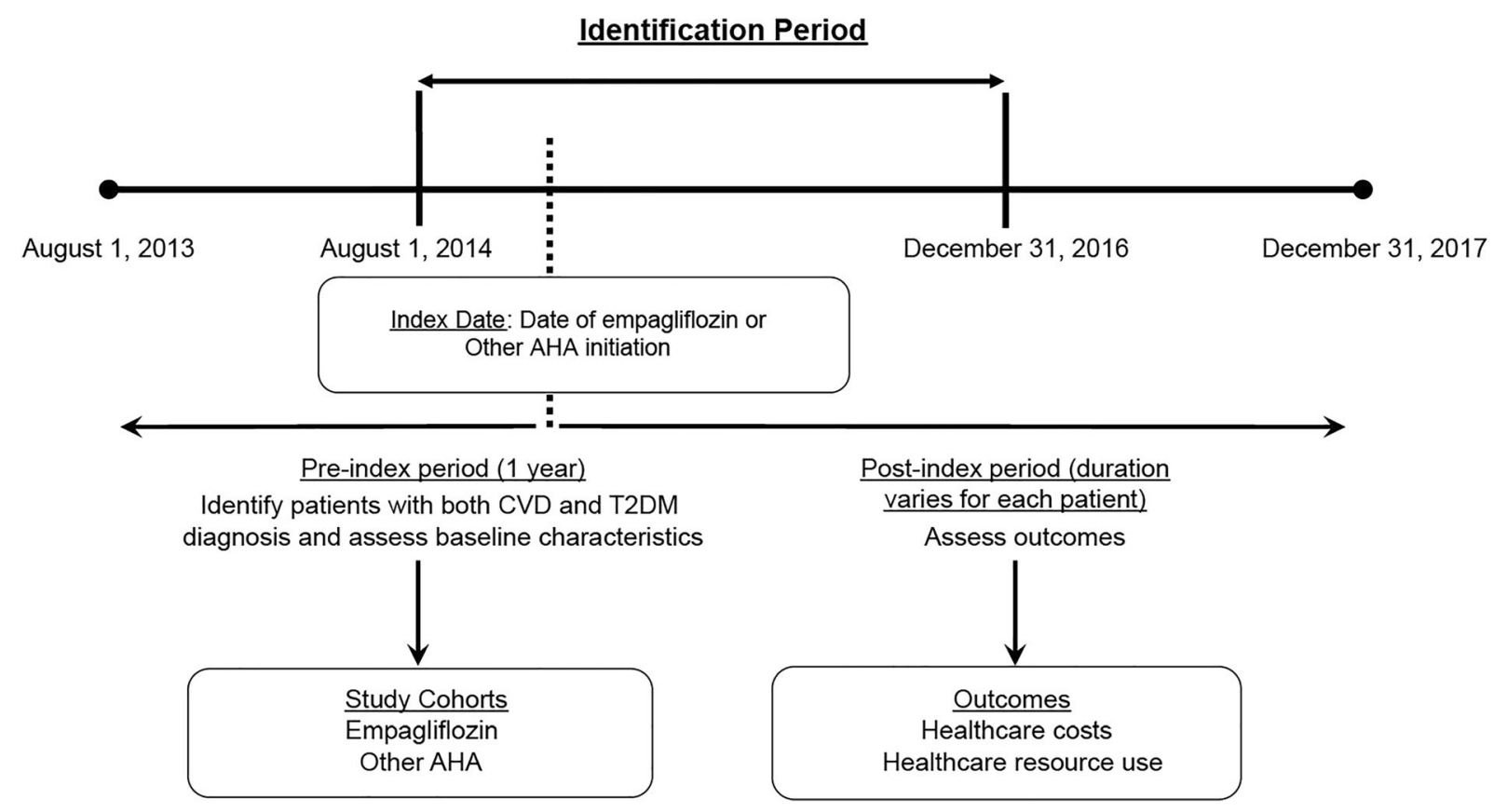

Fig. 1 Study design

\section{Study Population and Eligibility Criteria}

The primary study sample included adult patients with T2DM and CVD who newly initiated treatment with empagliflozin or other branded AHAs during the identification period. AHAs not belonging to the drug class of the study drugs (either empagliflozin or all other branded AHAs) were considered as standard of care and their use was allowed during the study period. Evidence of T2DM was ascertained by the presence of at least one non-diagnostic medical claim containing a primary or secondary diagnosis of T2DM (International Classification of Diseases, 9th Revision, Clinical Modification [ICD-9-CM]: 250.x0 or 250.x2; or ICD-10: E11) during the 12-month pre-index period. CVD was ascertained by the presence of at least one inpatient medical claim or at least two non-diagnostic outpatient medical claims occurring at least 1 month apart, but not separated by more than 12 months, containing a primary or secondary diagnosis for the same CVD condition, with the first claim occurring during the pre-index period. CVD conditions include the following: myocardial infarction (acute, chronic, and old), angina pectoris, heart failure, stroke, other ischemic disease, arrhythmias, cardiac arrest, atherosclerosis, peripheral vascular disease, arterial thrombosis and embolism, cardiomyopathy, conduction disorders, endocarditis, pericarditis, myocarditis, rheumatic heart disease and fever, and other heart disease. Patients were required to have continuous enrollment with medical and pharmacy benefits for the 12-month pre-index period and for at least the 2-month post-index period.

Patients who met the following criteria were excluded from the study:

1. Missing demographic information at baseline (age, gender, geographic region)

2. Had a diagnosis of pregnancy, gestational diabetes, secondary diabetes, type 1 diabetes mellitus, cancer, end-stage renal disease, human immunodeficiency virus, or transplant any time during the pre-index or post-index period

3. Had a non-commercial payer type on index date

4. Had received more than one branded AHA on index date

5. Had at least one claim for any branded AHA during the pre-index period (i.e., all these 
patients were naïve to empagliflozin and other branded AHAs in the comparison cohort)

\section{Study Outcomes}

The primary outcome of the study was all-cause total healthcare costs and included costs from all pharmacy and medical claims (regardless of diagnosis) during the post-index period. Other outcomes included all-cause HCRU, overall and by site of care.

Healthcare costs in this study represented actual reimbursements paid by health plans, plus any patient cost-sharing in the form of deductibles, copayments, and coinsurance for each medical or prescription encounter. For HCRU, the number of all-cause visits by care setting (inpatient, ED, physician office, and outpatient visits) and length of stay (LOS) per inpatient visit were assessed. Costs (after adjustment to 2017 US dollars) and HCRU were computed on a per patient per month (PPPM) basis. In addition, the risk (i.e., incidence) of an inpatient visit was also assessed and reported as the number of unique patients with an inpatient visit per 100 person-years (PYs).

\section{Statistical Analysis}

Descriptive statistics were used to characterize the study sample during the pre-index period and the outcomes during the post-index period. Patient baseline characteristics were compared using standardized differences (defined as the difference in means divided by the pooled standard deviation and multiplied by 100), where a difference of less than $10 \%$ was considered acceptable (or supported the assumption of balance) between the study cohorts.

The differences in costs between the study cohorts were evaluated using multivariate gamma regression models. The adjusted costs were predicted for patients in the empagliflozin and other branded AHA cohorts and the cost differences and 95\% CI were reported. Similarly, negative binomial regression models were used to compare HCRU (including LOS per inpatient visit) among the study cohorts and predicted values of HCRU are reported. The rate of inpatient visits per 100 PYs was compared using univariate Poisson regression models, and the risk of inpatient visits was analyzed using multivariate Cox proportional hazards models, and the results were reported as hazard ratios (HRs) with 95\% CIs. Pre-index characteristics included in the multivariate models were age; sex; region; health plan type; index year; Charlson comorbidity index (CCI); select comorbidities (hypertension, dyslipidemia, obesity, asthma, chronic obstructive pulmonary disease); indicator whether the index drug was an add-on therapy; number of CVD drug classes; number of antidiabetic drug classes; all-cause costs; allcause hospitalization; all-cause ED visit; endocrinologist visit; revascularization procedure; and type of CVD condition. All analyses were conducted using SAS $^{\circledR}$ version 9.4 (SAS Institute; Cary, NC, USA). An alpha level of 0.05 for statistical significance was used.

\section{Sensitivity Analysis}

In order to assess the robustness of the findings, a sensitivity analysis was conducted to evaluate whether the study results could be replicated in a broader population (i.e., including patients who used AHAs of interest during the pre-index period). For this analysis, the criterion that excluded patients on the basis of the presence of at least one claim for branded AHAs during the pre-index period was relaxed. Accordingly, only patients with claims for drugs belonging to the index drug class during the pre-index period were excluded (to ensure patients are treatmentnaïve to drugs belonging to the index drug class only). Costs and HCRU were compared among these updated study cohorts after controlling for pre-index AHA use and other baseline measures specified in the main analysis. 


\section{RESULTS}

\section{Overall Population}

\section{Baseline Characteristics}

A total of 13,563 patients were identified who had T2DM and comorbid CVD and received empagliflozin or other branded AHAs, and who were naïve to these index treatments (i.e., who did not receive these treatments during the preindex period). Of these identified patients, 441 and 13,122 patients were included in the empagliflozin and other branded AHA cohorts, respectively. In general, the empagliflozin cohort was younger and had a lower comorbidity burden during the pre-index period compared to the other branded AHAs cohort (Table 1). Compared to the other branded AHAs cohort, the number of antidiabetic drug classes received in the pre-index was higher in the empagliflozin cohort and the index drug was more likely to be an add-on therapy for the empagliflozin cohort. Patients in the empagliflozin cohort had lower all-cause hospitalizations and all-cause costs in the pre-index period. The type of CVD was generally similar across the study cohorts; however, the empagliflozin cohort had a lower proportion of patients with heart failure, stroke, arrhythmias, and cardiomyopathy (Table 1).

\section{Study Outcomes}

The average follow-up time was 7.4 months, with a longer follow-up time for the empagliflozin cohort vs. the other branded AHAs cohort $(8.0$ vs. 7.3 months; standardized difference $=16.6 \%$ ). During the post-index period, the unadjusted total all-cause total healthcare costs were significantly lower for the empagliflozin cohort vs. other branded AHAs cohort, with a mean cost difference of $-\$ 798$ PPPM $(p<0.001)$. After covariate adjustment, the adjusted total all-cause cost difference (mean - \$412 PPPM; 95\% CI - \$593, - \$214; $p<0.001)$ remained significantly lower for the empagliflozin cohort (Fig. 2). Among medical cost components, adjusted mean inpatient costs ( $\$ 475$ vs. $\$ 823 ; \Delta=-\$ 348, p=0.994$ ) and other outpatient/ancillary service costs $(\$ 375$ vs. $\$ 514 ; \Delta=-\$ 139, p<0.001)$ were the key components of lower PPPM all-cause medical costs for empagliflozin cohort vs. other branded AHAs cohort, although the difference in inpatient costs did not reach statistical significance (Fig. 3). No significant difference was observed in all-cause pharmacy costs $(\Delta=-\$ 31$, $p=0.554)$.

During the post-index period, the unadjusted total PPPM all-cause HCRU, across all settings of care, was also significantly lower for the empagliflozin cohort vs. other branded AHAs cohort $(p<0.001)$ (Table 2). After covariate adjustment, the mean PPPM all-cause visits in physician office and that in other outpatient settings were lower in the empagliflozin cohort compared to the other branded AHAs cohort $(p<0.001)$. Multivariate analyses for inpatient and ED visits were not evaluable, as the statistical models did not converge. Furthermore, the incidence rate of inpatient visits was significantly lower for the empagliflozin cohort vs. other branded AHA cohort (i.e., 9.9 vs. 18.8) per 100 PYs $(p=0.001)$, respectively. After adjustment for covariates, patients in the empagliflozin cohort had a 31\% lower risk of an inpatient visit compared to the other branded AHAs cohort; however, this difference in risk did not reach statistical significance (adjusted HR 0.69 ; 95\% CI $0.48,1.01 ; p=0.057$ ). While assessing LOS per inpatient visit, although numerically lower in the empagliflozin cohort, no significant differences were observed between the study cohorts (adjusted mean LOS, 5.5 days [empagliflozin] vs. 6.1 days [other branded AHAs]; $p=0.429$ ).

\section{Sensitivity Analysis Population}

\section{Baseline Characteristics}

After relaxing the criterion that excluded patients on the basis of presence of at least one claim for empagliflozin or other branded AHAs during the pre-index period, a total of 14,848 patients with T2DM and CVD who received other branded AHAs were identified. Of these, 1196 and 13,652 patients were included in the empagliflozin and other branded AHA cohorts, respectively. In general, the trends in baseline 
Table 1 Baseline characteristics

\begin{tabular}{|c|c|c|c|c|c|}
\hline \multirow[t]{2}{*}{ Characteristics } & \multicolumn{2}{|c|}{ Empagliflozin } & \multicolumn{2}{|c|}{ Other branded AHAs } & \multirow{2}{*}{$\begin{array}{l}\text { Standardized } \\
\text { difference }(\%)^{\mathrm{a}}\end{array}$} \\
\hline & \multicolumn{2}{|c|}{$n=441$} & \multicolumn{2}{|c|}{$n=13,122$} & \\
\hline \multicolumn{6}{|l|}{ Demographic } \\
\hline \multicolumn{6}{|c|}{ Age as of index (in years) } \\
\hline Mean (SD) & 56.9 & $(8.1)$ & 58.2 & $(9.0)$ & 15.2 \\
\hline Male, $n(\%)$ & 311 & $(70.5 \%)$ & 9084 & $(69.2 \%)$ & 2.8 \\
\hline \multicolumn{6}{|c|}{ Geographic region, $n(\%)$} \\
\hline East & 94 & $(21.3 \%)$ & 2636 & $(20.1 \%)$ & 3.0 \\
\hline Midwest & 83 & $(18.8 \%)$ & 2872 & $(21.9 \%)$ & 7.6 \\
\hline South & 234 & $(53.1 \%)$ & 6413 & $(48.9 \%)$ & 8.4 \\
\hline West & 30 & $(6.8 \%)$ & 1201 & $(9.2 \%)$ & 8.7 \\
\hline \multicolumn{6}{|l|}{ Plan type, $n(\%)$} \\
\hline HMO & 27 & $(6.1 \%)$ & 772 & $(5.9 \%)$ & 1.0 \\
\hline Indemnity & 3 & $(0.7 \%)$ & 280 & $(2.1 \%)$ & 12.4 \\
\hline $\mathrm{PPO} / \mathrm{POS}$ & 411 & $(93.2 \%)$ & 12,038 & $(91.7 \%)$ & 5.5 \\
\hline Unknown/other $^{\mathrm{b}}$ & 0 & $(0.0 \%)$ & 32 & $(0.2 \%)$ & 7.0 \\
\hline \multicolumn{6}{|l|}{ Index year, $n(\%)$} \\
\hline 2014 & 46 & $(10.4 \%)$ & 2173 & $(16.6 \%)$ & 18.0 \\
\hline 2015 & 173 & $(39.2 \%)$ & 5983 & $(45.6 \%)$ & 12.9 \\
\hline 2016 & 222 & $(50.3 \%)$ & 4966 & $(37.8 \%)$ & 25.4 \\
\hline \multicolumn{6}{|c|}{ Clinical characteristics during the pre-index period } \\
\hline \multicolumn{6}{|l|}{$\mathrm{CCI}^{\mathrm{c}}$} \\
\hline Mean (SD) & 1.0 & $(1.0)$ & 1.3 & $(1.3)$ & 27.2 \\
\hline \multicolumn{6}{|l|}{ CCI category, $n(\%)$} \\
\hline 0 & 171 & $(38.8 \%)$ & 4219 & $(32.2 \%)$ & 13.9 \\
\hline 1 & 166 & $(37.6 \%)$ & 4481 & $(34.2 \%)$ & 7.3 \\
\hline 2 & 67 & $(15.2 \%)$ & 2283 & $(17.4 \%)$ & 6.0 \\
\hline$\geq 3$ & 37 & $(8.4 \%)$ & 2139 & $(16.3 \%)$ & 24.2 \\
\hline \multicolumn{6}{|c|}{ Select comorbidities, $n(\%)$} \\
\hline Hypertension & 351 & $(79.6 \%)$ & 10,118 & $(77.1 \%)$ & 6.0 \\
\hline Dyslipidemia & 393 & $(89.1 \%)$ & 10,325 & $(78.7 \%)$ & 28.7 \\
\hline Obesity & 37 & $(8.4 \%)$ & 1556 & $(11.9 \%)$ & 11.5 \\
\hline
\end{tabular}


Table 1 continued

\begin{tabular}{|c|c|c|c|c|c|}
\hline \multirow[t]{2}{*}{ Characteristics } & \multirow{2}{*}{\multicolumn{2}{|c|}{$\begin{array}{l}\text { Empagliflozin } \\
n=441\end{array}$}} & \multirow{2}{*}{\multicolumn{2}{|c|}{$\begin{array}{l}\text { Other branded AHAs } \\
n=13,122\end{array}$}} & \multirow{3}{*}{$\begin{array}{l}\begin{array}{l}\text { Standardized } \\
\text { difference (\%) }\end{array} \\
7.2\end{array}$} \\
\hline & & & & & \\
\hline Asthma & 14 & $(3.2 \%)$ & 600 & $(4.6 \%)$ & \\
\hline COPD & 23 & $(5.2 \%)$ & 1002 & $(7.6 \%)$ & 9.9 \\
\hline Index drug was an add-on therapy, $n(\%)$ & 336 & $(76.2 \%)$ & 7617 & $(58.1 \%)$ & 39.3 \\
\hline \multicolumn{6}{|l|}{ Number of pre-index antidiabetic drug classes } \\
\hline Mean (SD) & 1.3 & $(0.7)$ & 1.1 & $(0.8)$ & 33.8 \\
\hline \multicolumn{6}{|l|}{ Number of pre-index antidiabetic drug classes, $n$ (\%) } \\
\hline 0 & 50 & $(11.3 \%)$ & 3483 & $(26.5 \%)$ & 39.5 \\
\hline 1 & 214 & $(48.5 \%)$ & 5664 & $(43.2 \%)$ & 10.8 \\
\hline 2 & 161 & $(36.5 \%)$ & 3613 & $(27.5 \%)$ & 19.3 \\
\hline$\geq 3$ & 16 & $(3.6 \%)$ & 362 & $(2.8 \%)$ & 4.9 \\
\hline \multicolumn{6}{|l|}{ Number of pre-index CVD-related drug classes, ${ }^{\mathrm{d}} n(\%)$} \\
\hline 0 & 12 & $(2.7 \%)$ & 1402 & $(10.7 \%)$ & 32.2 \\
\hline 1 & 36 & $(8.2 \%)$ & 989 & $(7.5 \%)$ & 2.3 \\
\hline 2 & 48 & $(10.9 \%)$ & 1699 & $(13.0 \%)$ & 6.4 \\
\hline$\geq 3$ & 345 & $(78.2 \%)$ & 9032 & $(68.8 \%)$ & 21.4 \\
\hline \multicolumn{6}{|l|}{ Established CVD conditions, $n(\%)$} \\
\hline Myocardial infarction & 62 & $(14.1 \%)$ & 2130 & $(16.2 \%)$ & 6.1 \\
\hline Angina & 48 & $(10.9 \%)$ & 1337 & $(10.2 \%)$ & 2.3 \\
\hline Heart failure & 43 & $(9.8 \%)$ & 2149 & $(16.4 \%)$ & 19.7 \\
\hline Stroke & 46 & $(10.4 \%)$ & 1808 & $(13.8 \%)$ & 10.3 \\
\hline Arrhythmias & 94 & $(21.3 \%)$ & 3661 & $(27.9 \%)$ & 15.3 \\
\hline Cardiac arrest & 0 & $(0.0 \%)$ & 49 & $(0.4 \%)$ & 8.7 \\
\hline Atherosclerosis and other ischemic heart disease & 266 & $(60.3 \%)$ & 7373 & $(56.2 \%)$ & 8.4 \\
\hline Peripheral vascular disease & 35 & $(7.9 \%)$ & 1134 & $(8.6 \%)$ & 2.6 \\
\hline Arterial thrombosis and embolism & 3 & $(0.7 \%)$ & 90 & $(0.7 \%)$ & 0.1 \\
\hline Cardiomyopathy & 22 & $(5.0 \%)$ & 1248 & $(9.5 \%)$ & 17.5 \\
\hline Conduction disorders & 17 & $(3.9 \%)$ & 680 & $(5.2 \%)$ & 6.4 \\
\hline Endocarditis, pericarditis, myocarditis & 50 & $(11.3 \%)$ & 1590 & $(12.1 \%)$ & 2.4 \\
\hline Rheumatic heart disease and fever & 11 & $(2.5 \%)$ & 345 & $(2.6 \%)$ & 0.9 \\
\hline Other heart disease & 22 & $(5.0 \%)$ & 929 & $(7.1 \%)$ & 8.8 \\
\hline Revascularization procedure & 107 & $(24.3 \%)$ & 3059 & $(23.3 \%)$ & 2.2 \\
\hline
\end{tabular}


Table 1 continued

\begin{tabular}{|c|c|c|c|c|c|}
\hline \multirow[t]{2}{*}{ Characteristics } & \multirow{2}{*}{\multicolumn{2}{|c|}{$\begin{array}{l}\text { Empagliflozin } \\
n=441\end{array}$}} & \multirow{2}{*}{\multicolumn{2}{|c|}{$\begin{array}{l}\text { Other branded AHAs } \\
n=13,122\end{array}$}} & \multirow{2}{*}{$\begin{array}{l}\text { Standardized } \\
\text { difference }(\%)^{a}\end{array}$} \\
\hline & & & & & \\
\hline Pre-index hospitalization, $n(\%)$ & 102 & $(23.1 \%)$ & 4833 & $(36.8 \%)$ & 30.2 \\
\hline Pre-index ED visit, $n(\%)$ & 136 & $(30.8 \%)$ & 4261 & $(32.5 \%)$ & 3.5 \\
\hline Pre-index endocrinologist visit, $n$ (\%) & 58 & $(13.2 \%)$ & 1339 & $(10.2 \%)$ & 9.2 \\
\hline \multicolumn{6}{|l|}{ Pre-index all-cause costs $(\$)$} \\
\hline Mean (SD) & $\$ 21,225$ & $(33,201)$ & $\$ 28,803$ & $(46,731)$ & 18.7 \\
\hline
\end{tabular}

Bold values with a standardized difference exceeding $10 \%$ indicates a significant imbalance between study cohorts

$A H A$ antihyperglycemic agent, $C C I$ Charlson comorbidity index, COPD chronic obstructive pulmonary disease, CVD cardiovascular disease, $E D$ emergency department, $H M O$ health maintenance organization, $P O S$ point of service, PPO preferred provider organization, $S D$ standard deviation, T2DM type 2 diabetes mellitus

${ }^{a}$ Standardized difference $=100 \times\left(x_{1}-x_{2}\right) / \sqrt{ }\left\{\left(s_{1}{ }^{2}+s_{2}{ }^{2}\right) / 2\right\}$, where $x_{1}$ is the mean of group $1, x_{2}$ is the mean of group 2 , $s_{1}$ is the standard deviation of group 1 , and $s_{2}$ is the standard deviation of group 2

${ }^{\mathrm{b}}$ Consumer-directed healthcare, unknown/missing

${ }^{c}$ Excludes diagnoses of T2DM

${ }^{\mathrm{d}}$ Includes cardiotonics, antianginal agents, beta-blocking agents, calcium channel blockers, antiarrhythmic agents, antihypertensives, diuretics, vasopressors, antihyperlipidemics, cardiovascular agents-misc., anticoagulants, platelet aggregation inhibitors, thrombolytic enzymes

characteristics were similar to those observed in the main analysis; however, both the number of antidiabetic drug classes and the magnitude of difference in the number antidiabetic drug classes received in the pre-index period between the study cohorts were higher (Table 3 ). The proportion of patients with endocrinologist visits in the pre-index period was higher in the empagliflozin cohort vs. the other branded AHAs cohort.

\section{Study Outcomes}

Results of sensitivity analysis were generally consistent with the findings from the main analysis. During the post-index period, unadjusted total all-cause total costs were significantly lower for the empagliflozin cohort vs. other branded AHAs cohort, with a mean cost difference of $-\$ 373$ PPPM $(p<0.01)$. After covariate adjustment, the adjusted total allcause cost difference (mean - \$268 PPPM; $95 \% \mathrm{CI}-\$ 435,-\$ 88 ; p<0.01)$ remained significantly lower for the empagliflozin cohort (Table 4). Among medical cost components, adjusted mean inpatient costs $(\$ 564$ vs. \$808 PPPM; $\Delta=-\$ 244, p=0.72$ ) and other outpatient/ancillary service costs $(\$ 418$ vs. $\$ 514$ PPPM; $\Delta=-\$ 96, p<0.01)$ were the key components of lower all-cause medical costs for empagliflozin cohort vs. the other branded AHAs cohort, although the difference in inpatient costs did not reach statistical significance. Furthermore, during the post-index period, the mean PPPM post-index all-cause visits in inpatient, ED, physician office, and other outpatient settings were lower in the empagliflozin cohort compared to the other branded AHAs cohort (Table $4 ; p<0.001$ for all comparisons between cohorts, where evaluable).

\section{DISCUSSION}

Since 2008, the US Food and Drug Administration (FDA) has required drug companies to provide CV safety data for all new AHAs [23]. The EMPA-REG OUTCOME trial was a landmark study that proved CV safety and demonstrated 


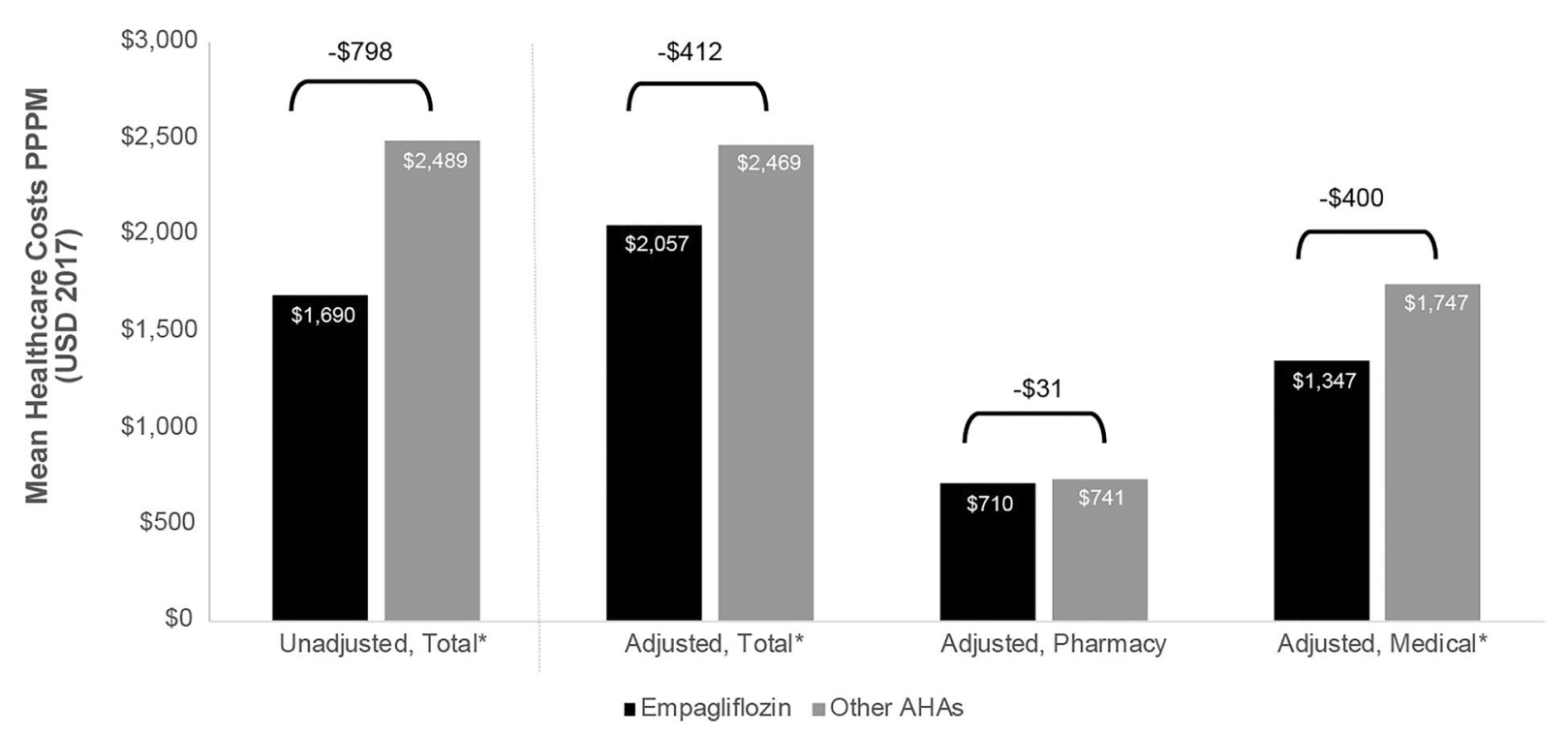

Fig. 2 All-cause healthcare costs (PPPM) during the post-index period. AHA antihyperglycemic agent, PPPM per patient per month, USD United States dollars. ${ }^{*} p<0.001$

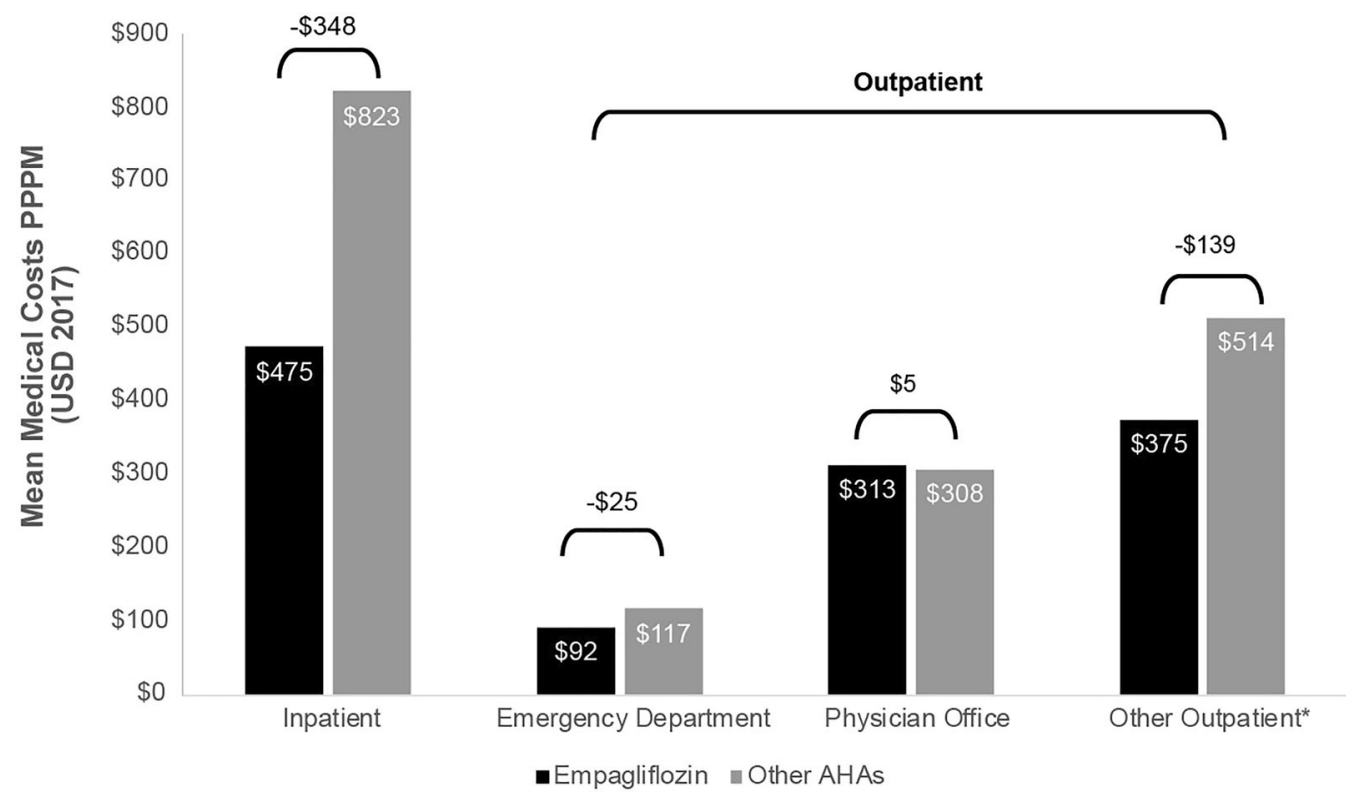

Fig. 3 All-cause medical costs (PPPM) during the post-index period. AHA antihyperglycemic agent, PPPM per patient per month, USD United States dollars. ${ }^{*} p<0.001$

cardioprotective benefits of an AHA. Results of that study demonstrated the efficacy of empagliflozin in reducing the risk of three-point MACE, HHF, and CV and all-cause death [7]. Those findings were corroborated by interim results from an ongoing real-world study
(EMPRISE), which demonstrated a lower risk of HHF with empagliflozin vs. DPP4i in patients with and without baseline CVD treated in routine clinical practice [15]. Furthermore, results from another real-world study, CVD-REAL, a propensity-matched observational study, also 
Table 2 All-cause healthcare resource utilization (per patient per month) during the post-index period

\begin{tabular}{|c|c|c|c|c|c|}
\hline \multirow[t]{2}{*}{ Outcomes } & \multicolumn{2}{|c|}{ Empagliflozin } & \multicolumn{2}{|c|}{ Other branded AHAs } & \multirow[t]{2}{*}{$p$ value } \\
\hline & $n=4$ & & $n=1$ & & \\
\hline \multicolumn{6}{|l|}{ Inpatient visits } \\
\hline Unadjusted, mean (SD) & 0.012 & $(0.053)$ & 0.025 & $(0.102)$ & $<0.0001$ \\
\hline Adjusted, mean (95\% CI) & NR & & NR & & NR \\
\hline \multicolumn{6}{|l|}{ Outpatient visits } \\
\hline \multicolumn{6}{|l|}{$\mathrm{ED}$} \\
\hline Unadjusted, mean (SD) & 0.035 & $(0.108)$ & 0.049 & $(0.171)$ & 0.0096 \\
\hline Adjusted, mean $(95 \% \mathrm{CI})$ & NR & & NR & & NR \\
\hline \multicolumn{6}{|l|}{ Physician office } \\
\hline Unadjusted, mean (SD) & 0.875 & $(1.000)$ & 1.162 & $(1.241)$ & $<0.0001$ \\
\hline Adjusted, mean $(95 \% \mathrm{CI})$ & 0.918 & $(0.842,1.000)$ & 1.082 & $(1.067,1.098)$ & 0.0002 \\
\hline \multicolumn{6}{|l|}{ Other outpatient } \\
\hline Unadjusted, mean (SD) & 0.411 & $(0.534)$ & 0.732 & $(1.300)$ & $<0.0001$ \\
\hline Adjusted, mean (95\% CI) & 0.446 & $(0.391,0.509)$ & 0.621 & $(0.608,0.634)$ & $<0.0001$ \\
\hline
\end{tabular}

Bold $p$ values indicate statistical significance $(p<0.05)$

$A H A$ antihyperglycemic agent, $C I$ confidence interval, $E D$ emergency department, $N R$ not reported (multivariable models did not converge; hence, we were unable to obtain adjusted estimates), $S D$ standard deviation

showed a lower risk of HHF, all-cause death, and composite of HHF with the use of the SGLT2i compared to other AHAs [12, 13].

While empagliflozin has proven clinical efficacy in improving CV outcomes in patients with T2DM and comorbid CVD, there are limited data on its impact on economic outcomes compared to standard of care. The current study sought to assess the impact of empagliflozin on costs and HCRU relative to other branded AHAs. Results from this study demonstrated that the PPPM all-cause total healthcare costs were significantly lower for patients initiating empagliflozin vs. other branded AHAs. Among medical cost components, mean inpatient costs and other outpatient/ancillary service costs were the key components of lower PPPM all-cause medical costs for the empagliflozin cohort. These findings were substantiated by the results for the HCRU analysis, where the PPPM all-cause total HCRU, across all settings of care, were also lower for patients initiating empagliflozin vs. other branded AHAs.

This study is the first to evaluate the cost implications of initiating empagliflozin vs. other branded AHAs in patients with T2DM and CVD. Our study results are aligned with evidence from previous studies that demonstrated the cost savings associated with the use of SGLT2is in this patient population [24-27]. Using a targeted optimization model, Schneeweiss et al. assessed the medical savings expected with increased utilization of SGLT2i vs. sulfonylureas among commercially insured patients with T2DM and a high risk of CVD, based on prior hospitalization for CVD ( $n=1967)$. With $10 \%$ of eligible patients receiving an SGLT2i for a mean of 6 months, the total medical plan savings were estimated at $\$ 1.6$ million, largely a result of avoiding HHF; total medical plans savings were predicted to increase to $\$ 8$ million and $\$ 10.9$ million if the percentage of patients receiving SGLT2i 
Table 3 Baseline characteristics (sensitivity analysis)

\begin{tabular}{|c|c|c|c|c|c|}
\hline \multirow{3}{*}{$\begin{array}{l}\text { Characteristics } \\
\text { Demographic }\end{array}$} & \multirow{2}{*}{\multicolumn{2}{|c|}{$\begin{array}{l}\text { Empagliflozin } \\
n=1196\end{array}$}} & \multirow{2}{*}{\multicolumn{2}{|c|}{$\begin{array}{l}\text { Other branded AHAs } \\
n=13,652\end{array}$}} & \multirow{3}{*}{$\begin{array}{l}\text { Standardized } \\
\text { difference }(\%)^{\mathrm{a}}\end{array}$} \\
\hline & & & & & \\
\hline & & & \\
\hline \multicolumn{6}{|c|}{ Age as of index (in years) } \\
\hline Mean (SD) & 57.4 & $(7.9)$ & 58.2 & (8.9) & 9.0 \\
\hline Male, $n(\%)$ & 862 & $(72.1 \%)$ & 9434 & $(69.1 \%)$ & 6.5 \\
\hline \multicolumn{6}{|c|}{ Geographic region, $n(\%)$} \\
\hline East & 271 & $(22.7 \%)$ & 2734 & $(20.0 \%)$ & 6.4 \\
\hline Midwest & 236 & $(19.7 \%)$ & 2983 & $(21.9 \%)$ & 5.2 \\
\hline South & 587 & $(49.1 \%)$ & 6708 & $(49.1 \%)$ & 0.1 \\
\hline West & 102 & $(8.5 \%)$ & 1227 & $(9.0 \%)$ & 1.6 \\
\hline \multicolumn{6}{|l|}{ Plan type, $n(\%)$} \\
\hline $\mathrm{HMO}$ & 79 & $(6.6 \%)$ & 794 & $(5.8 \%)$ & 3.3 \\
\hline Indemnity & 13 & $(1.1 \%)$ & 288 & $(2.1 \%)$ & 8.2 \\
\hline $\mathrm{PPO} / \mathrm{POS}$ & 1103 & $(92.2 \%)$ & 12,533 & $(91.8 \%)$ & 1.6 \\
\hline Unknown/other ${ }^{\mathrm{b}}$ & 1 & $(0.1 \%)$ & 37 & $(0.3 \%)$ & 4.5 \\
\hline \multicolumn{6}{|l|}{ Index year, $n(\%)$} \\
\hline 2014 & 138 & $(11.5 \%)$ & 2605 & $(19.1 \%)$ & 21.1 \\
\hline 2015 & 479 & $(40.1 \%)$ & 6081 & $(44.5 \%)$ & 9.1 \\
\hline 2016 & 579 & $(48.4 \%)$ & 4966 & $(36.4 \%)$ & 24.5 \\
\hline \multicolumn{6}{|c|}{ Clinical characteristics during pre-index period } \\
\hline \multicolumn{6}{|l|}{$\mathrm{CCI}^{\mathrm{c}}$} \\
\hline Mean (SD) & 1.0 & $(1.1)$ & 1.3 & $(1.3)$ & 22.3 \\
\hline \multicolumn{6}{|c|}{$\mathrm{CCI}^{\mathrm{c}}$ category, $n(\%)$} \\
\hline 0 & 474 & $(39.6 \%)$ & 4391 & $(32.2 \%)$ & 15.6 \\
\hline 1 & 413 & $(34.5 \%)$ & 4667 & $(34.2 \%)$ & 0.7 \\
\hline 2 & 182 & $(15.2 \%)$ & 2373 & $(17.4 \%)$ & 5.9 \\
\hline$\geq 3$ & 127 & $(10.6 \%)$ & 2221 & $(16.3 \%)$ & 16.6 \\
\hline \multicolumn{6}{|c|}{ Select comorbidities, $n$ (\%) } \\
\hline Hypertension & 940 & $(78.6 \%)$ & 10,521 & $(77.1 \%)$ & 3.7 \\
\hline Dyslipidemia & 1079 & $(90.2 \%)$ & 10,807 & $(79.2 \%)$ & 31.1 \\
\hline Obesity & 124 & $(10.4 \%)$ & 1651 & $(12.1 \%)$ & 5.5 \\
\hline Asthma & 42 & $(3.5 \%)$ & 633 & $(4.6 \%)$ & 5.7 \\
\hline
\end{tabular}


Table 3 continued

\begin{tabular}{|c|c|c|c|c|c|}
\hline \multirow[t]{2}{*}{ Characteristics } & \multirow{2}{*}{\multicolumn{2}{|c|}{$\begin{array}{l}\text { Empagliflozin } \\
n=1196\end{array}$}} & \multirow{2}{*}{\multicolumn{2}{|c|}{$\begin{array}{l}\text { Other branded AHAs } \\
n=13,652\end{array}$}} & \multirow{3}{*}{$\begin{array}{l}\begin{array}{l}\text { Standardized } \\
\text { difference (\%) }\end{array} \\
14.3\end{array}$} \\
\hline & & & & & \\
\hline COPD & 50 & $(4.2 \%)$ & 1029 & $(7.5 \%)$ & \\
\hline Index drug was an add-on therapy, $n(\%)$ & 1050 & $(87.8 \%)$ & 8030 & $(58.8 \%)$ & 69.3 \\
\hline \multicolumn{6}{|l|}{ Number of pre-index antidiabetic drug classes } \\
\hline Mean $(\mathrm{SD})$ & 2.1 & $(1.1)$ & 1.1 & $(0.9)$ & 97.1 \\
\hline \multicolumn{6}{|l|}{ Number of pre-index antidiabetic drug classes, $n$ (\%) } \\
\hline 0 & 50 & $(4.2 \%)$ & 3483 & $(25.5 \%)$ & 62.9 \\
\hline 1 & 335 & $(28.0 \%)$ & 5746 & $(42.1 \%)$ & 29.8 \\
\hline 2 & 444 & $(37.1 \%)$ & 3805 & $(27.9 \%)$ & 19.8 \\
\hline$\geq 3$ & 367 & $(30.7 \%)$ & 618 & $(4.5 \%)$ & 73.1 \\
\hline \multicolumn{6}{|c|}{ Number of pre-index CVD-related drug classes, ${ }^{\mathrm{d}} n$ (\%) } \\
\hline 0 & 25 & $(2.1 \%)$ & 1413 & $(10.4 \%)$ & 34.7 \\
\hline 1 & 68 & $(5.7 \%)$ & 1017 & $(7.5 \%)$ & 7.1 \\
\hline 2 & 155 & $(13.0 \%)$ & 1763 & $(12.9 \%)$ & 0.1 \\
\hline$\geq 3$ & 948 & $(79.3 \%)$ & 9459 & $(69.3 \%)$ & 23.0 \\
\hline \multicolumn{6}{|l|}{ Established CVD conditions, $n(\%)$} \\
\hline Myocardial infarction & 162 & $(13.6 \%)$ & 2199 & $(16.1 \%)$ & 7.2 \\
\hline Angina & 124 & $(10.4 \%)$ & 1384 & $(10.1 \%)$ & 0.8 \\
\hline Heart failure & 127 & $(10.6 \%)$ & 2230 & $(16.3 \%)$ & 16.8 \\
\hline Stroke & 119 & $(10.0 \%)$ & 1881 & $(13.8 \%)$ & 11.9 \\
\hline Arrhythmias & 266 & $(22.2 \%)$ & 3798 & $(27.8 \%)$ & 12.9 \\
\hline Cardiac arrest & 2 & $(0.2 \%)$ & 49 & $(0.4 \%)$ & 3.7 \\
\hline Atherosclerosis and other ischemic heart disease & 741 & $(62.0 \%)$ & 7690 & $(56.3 \%)$ & 11.5 \\
\hline Peripheral vascular disease & 89 & $(7.4 \%)$ & 1184 & $(8.7 \%)$ & 4.5 \\
\hline Arterial thrombosis and embolism & 8 & $(0.7 \%)$ & 92 & $(0.7 \%)$ & 0.1 \\
\hline Cardiomyopathy & 86 & $(7.2 \%)$ & 1294 & $(9.5 \%)$ & 8.3 \\
\hline Conduction disorders & 43 & $(3.6 \%)$ & 702 & $(5.1 \%)$ & 7.6 \\
\hline Endocarditis, pericarditis, myocarditis & 116 & $(9.7 \%)$ & 1656 & $(12.1 \%)$ & 7.8 \\
\hline Rheumatic heart disease and fever & 15 & $(1.3 \%)$ & 353 & $(2.6 \%)$ & 9.7 \\
\hline Other heart disease & 54 & $(4.5 \%)$ & 953 & $(7.0 \%)$ & 10.6 \\
\hline Revascularization procedure & 273 & $(22.8 \%)$ & 3182 & $(23.3 \%)$ & 1.1 \\
\hline
\end{tabular}


Table 3 continued

\begin{tabular}{|c|c|c|c|c|c|}
\hline \multirow[t]{2}{*}{ Characteristics } & \multirow{2}{*}{\multicolumn{2}{|c|}{$\begin{array}{l}\text { Empagliflozin } \\
n=1196\end{array}$}} & \multirow{2}{*}{\multicolumn{2}{|c|}{$\begin{array}{l}\text { Other branded AHAs } \\
n=13,652\end{array}$}} & \multirow{2}{*}{$\begin{array}{l}\text { Standardized } \\
\text { difference }(\%)^{a}\end{array}$} \\
\hline & & & & & \\
\hline Pre-index hospitalization, $n$ (\%) & 282 & $(23.6 \%)$ & 5017 & $(36.8 \%)$ & 29.0 \\
\hline Pre-index ED visit, $n(\%)$ & 340 & $(28.4 \%)$ & 4429 & $(32.4 \%)$ & 8.7 \\
\hline Pre-index endocrinologist visit, $n$ (\%) & 268 & $(22.4 \%)$ & 1450 & $(10.6 \%)$ & 32.1 \\
\hline \multicolumn{6}{|l|}{ Pre-index all-cause costs $(\$)$} \\
\hline Mean (SD) & $\$ 24,514$ & $(30,299)$ & $\$ 28,918$ & $(46,545)$ & 11.2 \\
\hline
\end{tabular}

Bold values with a standardized difference exceeding $10 \%$ indicates a significant imbalance between study cohorts $A H A$ antihyperglycemic agent, $C C I$ Charlson comorbidity index, COPD chronic obstructive pulmonary disease, CVD cardiovascular disease, $E D$ emergency department, $H M O$ health maintenance organization, $P O S$ point of service, $P P O$ preferred provider organization, $S D$ standard deviation, T2DM type 2 diabetes mellitus

${ }^{2}$ Standardized difference $=100 \times\left(x_{1}-x_{2}\right) / \sqrt{ }\left\{\left(s_{1}{ }^{2}+s_{2}{ }^{2}\right) / 2\right\}$, where $x_{1}$ is the mean of group $1, x_{2}$ is the mean of group 2 , $s_{1}$ is the standard deviation of group 1 , and $s_{2}$ is the standard deviation of group 2

${ }^{\mathrm{b}}$ Consumer-directed healthcare, unknown/missing

${ }^{c}$ Excludes diagnoses of T2DM

${ }^{\mathrm{d}}$ Includes cardiotonics, antianginal agents, beta-blocking agents, calcium channel blockers, antiarrhythmic agents, antihypertensives, diuretics, vasopressors, antihyperlipidemics, cardiovascular agents-misc., anticoagulants, platelet aggregation inhibitors, thrombolytic enzymes

increased to $75 \%$ or the duration of treatment increased by 2 months, respectively. Chen et al. compared HHF costs and total healthcare costs among patients with T2DM and CVD who initiated canagliflozin vs. a non-SGLT2i AHA in OBSERVE-4D, a real-world observational study. The authors found that the reduction in risk of HHF for patients with T2DM and concomitant CVD who were treated with canagliflozin was accompanied by a significant reduction in both HHF costs and total healthcare costs. Najafzadeh et al. compared costs and HCRU between empagliflozin and DPP4i users in a propensitymatched cohort study (EMPRISE study) [26, 27]. Results from that study showed a trend toward lower inpatient and outpatient costs and significantly lower HCRU among patients initiating empagliflozin compared to DPP4i.

Selection of a cost-effective intervention is a critical consideration for healthcare decision makers while managing patients with T2DM and comorbid CVD, especially because the prevalence of T2DM and comorbid CVD continues to rise [28]. Findings from this study quantify the beneficial effects of empagliflozin in terms of costs and HCRU in patients with
T2DM and CVD, and provide healthcare decision makers with much needed economic data that can be integrated with clinical evidence to aid formulary reviews and coverage decisions.

\section{Limitations}

Several limitations of our current study should be considered when interpreting the results. This study presents results for patients covered by commercial health insurance plans, a population which tends to be younger and healthier; hence, these results may not be generalizable to older patients (65 years and older) covered through either Medicare or Medicaid. The results of this study may be confounded by some important, but unavailable, clinical factors, such as glycemic control parameters (like hemoglobin A1c), physical activity, weight, and smoking status. Furthermore, since comorbidities and other clinical variables were captured in this study through diagnosis and procedure codes, measurement errors may exist if there were inconsistencies in the reported codes. Finally, given the retrospective cohort design, 
Table 4 All-cause healthcare costs and resource utilization (per patient per month) during the post-index period (sensitivity analysis)

\begin{tabular}{|c|c|c|c|c|c|}
\hline \multirow[t]{2}{*}{ Outcomes } & \multicolumn{2}{|c|}{ Empagliflozin } & \multicolumn{2}{|c|}{ Other branded AHAs } & \multirow[t]{2}{*}{$p$ value } \\
\hline & \multicolumn{2}{|c|}{$n=1196$} & \multicolumn{2}{|c|}{$n=13,652$} & \\
\hline \multicolumn{6}{|l|}{ Costs } \\
\hline \multicolumn{6}{|l|}{ Total } \\
\hline Unadjusted, mean (SD) & $\$ 2120$ & $(3662)$ & $\$ 2493$ & $(5480)$ & 0.0015 \\
\hline Adjusted, mean & $\$ 2360$ & & $\$ 2628$ & & \\
\hline Adjusted mean difference $(95 \% \mathrm{CI})$ & $-\$ 268$ & $(-435,-88)$ & & & 0.0045 \\
\hline \multicolumn{6}{|l|}{ Pharmacy } \\
\hline Unadjusted, mean (SD) & $\$ 988$ & $(741)$ & $\$ 759$ & $(1035)$ & $<0.0001$ \\
\hline Adjusted, mean (95\% CI) & $\$ 766$ & & $\$ 785$ & & \\
\hline Adjusted mean difference $(95 \% \mathrm{CI})$ & $-\$ 18$ & $(-55,20)$ & & & 0.3395 \\
\hline \multicolumn{6}{|l|}{ Medical } \\
\hline Unadjusted, mean (SD) & $\$ 1132$ & $(3558)$ & $\$ 1734$ & $(5723)$ & $<0.0001$ \\
\hline Adjusted, mean (95\% CI) & $\$ 1570$ & & $\$ 1899$ & & \\
\hline Adjusted mean difference $(95 \% \mathrm{CI})$ & $-\$ 329$ & $(-496,-142)$ & & & 0.0006 \\
\hline \multicolumn{6}{|l|}{ HCRU } \\
\hline \multicolumn{6}{|l|}{ Inpatient visits } \\
\hline Unadjusted, mean (SD) & 0.013 & $(0.061)$ & 0.047 & $(0.137)$ & $<0.0001$ \\
\hline Adjusted, mean (95\% CI) & NR & & NR & & NR \\
\hline \multicolumn{6}{|l|}{ Outpatient visits } \\
\hline \multicolumn{6}{|l|}{$\mathrm{ED}$} \\
\hline Unadjusted, mean (SD) & 0.032 & $(0.104)$ & 0.049 & $(0.169)$ & $<0.0001$ \\
\hline Adjusted, mean $(95 \% \mathrm{CI})$ & NR & & NR & & NR \\
\hline \multicolumn{6}{|l|}{ Physician office } \\
\hline Unadjusted, mean (SD) & 0.981 & $(1.068)$ & 1.165 & $(1.244)$ & $<0.0001$ \\
\hline Adjusted, mean $(95 \% \mathrm{CI})$ & 0.940 & $(0.884,1.000)$ & 1.087 & $(1.072,1.103)$ & $<0.0001$ \\
\hline \multicolumn{6}{|l|}{ Other outpatient } \\
\hline Unadjusted, mean (SD) & 0.467 & $(0.609)$ & 0.727 & $(1.289)$ & $<0.0001$ \\
\hline Adjusted, mean (95\% CI) & 0.473 & $(0.431,0.519)$ & 0.618 & $(0.605,0.631)$ & $<0.0001$ \\
\hline
\end{tabular}

Bold $p$ values indicate statistical significance $(p<0.05)$

$A H A$ antihyperglycemic agent, $C I$ confidence interval, $E D$ emergency department, $H C R U$ healthcare resource utilization, $N R$ not reported (multivariable models did not converge; hence, we were unable to obtain adjusted estimates), $S D$ standard deviation 
the findings from this study only demonstrate an association and not causation.

\section{CONCLUSIONS}

Although there is a strong clinical evidence base that highlights the cardioprotective benefits of AHAs, there are limited studies that assessed whether these benefits translate into cost savings. Given that the incremental economic burden of managing patients with T2DM and CVD is substantial, there is a need to identify treatments that have supplemental economic benefits. This retrospective cohort study fulfills that unmet need and demonstrates that initiating empagliflozin vs. other branded AHAs was associated with lower all-cause costs among patients diagnosed with T2DM and CVD. The results complement the established clinical benefit profile of empagliflozin and can guide healthcare decision makers during therapy selection to decrease total cost of care for the healthcare system.

\section{ACKNOWLEDGEMENTS}

Funding. Funding for this research study and the journal's Rapid Service Fee was provided by Boehringer Ingelheim Pharmaceuticals, Inc.

Medical Writing, Editorial, and Other Assistance. The authors would like to acknowledge Kristin Hennenfent, PharmD, MBA, BCOP, BCPS, Xcenda LLC, for her medical writing contribution.

Authorship. All named authors meet the International Committee of Medical Journal Editors (ICMJE) criteria for authorship for this article, take responsibility for the integrity of the work as a whole, and have given their approval for this version to be published.

Author Contributions. Study concept and design: all authors; statistical analyses: AR, EF; critical review and interpretation of data: all authors. Drafting of manuscript: KH. Critical review, revision, and approval of manuscript: all authors.

Prior Presentation. This work was presented as a poster at the American Heart Association's Quality of Care and Outcomes Research 2019 Scientific Sessions.

Disclosures. Aditya Raju, Dana Stafkey-Mailey, and Eileen Farrelly have disclosed that they are employees of Xcenda LLC, which has received research funding from Boehringer Ingelheim Pharmaceuticals, Inc. for the conduct of this study and for the preparation of this manuscript. Pratik Pimple and Sharash Shetty have disclosed that they are employees of Boehringer Ingelheim Pharmaceuticals, Inc.

Compliance with Ethics Guidelines. This was a non-interventional study that used anonymized and HIPAA-compliant data from a commercially available administrative claims database (https://www.iqvia.com/library/factsheets/iqvia-pharmetrics-plus). Institutional review board approval was not required as individually identifiable data were not collected or used during the conduct of this study.

Data Availability. Data for this study were made available through a third-party license from IQVIA, a commercial data provider in the USA. Data are proprietary to IQVIA, and further release of the dataset is not permitted due to the data use agreement. All data generated or analyzed during this study are summarized and included in this published article.

Open Access. This article is licensed under a Creative Commons Attribution-NonCommercial 4.0 International License, which permits any non-commercial use, sharing, adaptation, distribution and reproduction in any medium or format, as long as you give appropriate credit to the original author(s) and the source, provide a link to the Creative Commons licence, and indicate if changes were made. The images or other third party material in this article are included in the article's Creative Commons licence, unless indicated otherwise in a credit 
line to the material. If material is not included in the article's Creative Commons licence and your intended use is not permitted by statutory regulation or exceeds the permitted use, you will need to obtain permission directly from the copyright holder. To view a copy of this licence, visit http://creativecommons.org/licenses/by$\mathrm{nc} / 4.0 /$.

\section{REFERENCES}

1. American Diabetes Association (ADA). Economic costs of diabetes in the U.S. in 2017. Diabetes Care. 2018;41(5):917-28.

2. American Heart Association (AHA) 2015. Cardiovascular diseases \& diabetes. 2015. Accessed 1 Nov 2018. http://www.heart.org/HEARTORG/ Conditions/More/Diabetes/WhyDiabetesMatters/ Cardiovascular-Disease-Diabetes_UCM_313865_ Article.jsp/\#.WT7R9HeZPow.

3. American Diabetes Association (ADA). Cardiovascular disease and risk management. Diabetes Care. 2017;40(Suppl1):S75-87.

4. Mehta S, Ghosh S, Sander S, et al. Differences in allcause health care utilization and costs in a type 2 diabetes mellitus population with and without a history of cardiovascular disease. J Manag Care Spec Pharm. 2018;24(3):280-90.

5. Einarson TR, Acs A, Ludwig C, Panton UH. Economic burden of cardiovascular disease in type 2 diabetes: a systematic review. Value Health. 2018;21(7):881-90.

6. Kramer CK, Zinman B. Sodium-glucose cotransporter-2 (SGLT-2) inhibitors and the treatment of type 2 diabetes. Annu Rev Med. 2019;70:323-34.

7. Zinman B, Wanner C, Lachin JM, et al. Empagliflozin, cardiovascular outcomes, and mortality in type 2 diabetes. N Engl J Med. 2015;373(22): 2117-28.

8. Neal B, Perkovic V, Mahaffey KW, et al. Canagliflozin and cardiovascular and renal events in type 2 diabetes. N Engl J Med. 2017;377:644-57.

9. Schnell O, Standl E, Catrinoiu D, et al. Report from the 3rd Cardiovascular Outcome Trial (CVOT) Summit of the Diabetes \& Cardiovascular Disease (D\&CVD) EASD Study Group. Cardiovasc Diabetol. 2018;17(1):30.
10. Wiviott SD, Raz I, Bonaca MP, et al. Dapagliflozin and cardiovascular outcomes in type 2 diabetes. N Engl J Med. 2018;380:347-57.

11. Verma S, McMurray JJV. SGLT2 inhibitors and mechanisms of cardiovascular benefit: a state-ofthe-art review. Diabetologia. 2018;61(10):2108-17.

12. Kosiborod M, Cavender MA, Fu AZ, et al. CVD-REAL Investigators and Study Group. Lower risk of heart failure and death in patients initiated on sodiumglucose cotransporter-2 inhibitors versus other glucose-lowering drugs: the CVD-REAL (comparative effectiveness of cardiovascular outcomes in new users of sodium-glucose cotransporter- 2 inhibitors). Circulation. 2018;136(3):249-259.

13. Saad M. Sodium-glucose cotransporter-2 inhibitors and cardiovascular outcomes: insights from the CVD-REAL study. Ann Transl Med. 2018;6(3):55.

14. Mahaffey KW, Neal B, Perkovic V, et al. CANVAS Program Collaborative Group. Canagliflozin for primary and secondary prevention of cardiovascular events: results from the CANVAS program (Canagliflozin Cardiovascular Assessment Study). Circulation. 2018;137(4):323-34.

15. Patorno E, Pawar A, Franklin J, et al. Empagliflozin and the risk of heart failure hospitalization in routine clinical care: a first analysis from the EMPRISE study. Circulation. 2019;139:2822-30.

16. Zinman B, Inzucchi SE, Lachin JM, et al. Rationale, design, and baseline characteristics of a randomized, placebo-controlled cardiovascular outcome trial of empagliflozin (EMPA-REG OUTCOME ${ }^{\mathrm{TM}}$ ). Cardiovasc Diabetol. 2014;13:102.

17. Gautam S, Agiro A, Barron J, Power T, Weisman H, White J. Heart failure hospitalization risk associated with use of two classes of oral antidiabetic medications: an observational, real-world analysis. Cardiovasc Diabetol. 2017;16:93.

18. Patorno E, Goldfine A, Schneeweiss S, et al. Cardiovascular outcomes associated with canagliflozin versus other non-gliflozin antidiabetic drugs: population based cohort study. BMJ. 2018;360:k119.

19. Ryan P, Buse J, Schuemie M, et al. Comparative effectiveness of canagliflozin, SGLT2 inhibitors and non-SGLT2 inhibitors on the risk of hospitalization for heart failure and amputation in patients with type 2 diabetes mellitus: a real-world meta-analysis of 4 observational databases (OBSERVE-4D). Diabetes Obes Metab. 2018;20(11):2585-97.

20. Udell JA, Yuan Z, Rush T, Sicignano NM, Galitz M, Rosenthal N. Cardiovascular outcomes and risks after initiation of a sodium glucose cotransporter 2 inhibitor: results from the EASEL population-based 
cohort study (Evidence for Cardiovascular Outcomes with Sodium Glucose Cotransporter 2 Inhibitors in the Real World). Circulation. 2018;137(14):1450-9.

21. Das SR, Everett BM, Birtcher KK, et al. 2018 ACC expert consensus decision pathway on novel therapies for cardiovascular risk reduction in patients with type 2 diabetes and atherosclerotic cardiovascular disease: a report of the American College of Cardiology Task Force on Expert Consensus Decision Pathways. J Am Coll Cardiol. 2018;72:3200-23.

22. American Diabetes Association. Pharmacologic approaches to glycemic treatment: standards of medical care in diabetes-2019. Diabetes Care. 2019;42(Suppl 1):S90-102.

23. Food and Drug Administration (FDA). Guidance for industry: diabetes mellitus-evaluating cardiovascular risk in new antidiabetic therapies to treat type 2 diabetes. 2008. Accessed 21 May 2019. https://www.federalregister.gov/documents/2008/ 12/19/E8-30086/guidance-for-industry-on-diabetesmellitus-evaluating-cardiovascular-risk-in-newantidiabetic

24. Schneeweiss S, Eapen S, Jan S. Quantifying the opportunity to reduce total cost of care through targeted medication prioritization and adherence improvement in diabetes. Value Health. 2019;22(Suppl 2):S39 (Abstract:DM3).

25. Chen YW, Voelker J, Tunceli O, Pericone C, Bookhart B, Durkin M. Real-world comparison of hospitalization costs for heart failure in type 2 diabetes mellitus patients with cardiovascular disease treated with canagliflozin versus other antihyperglycemic agents. Presented at ISPOR 2019; May 18-22, 2019. New Orleans; 2019.

26. Najafzadeh M, Pawar A, Déruaz Luyet A, et al. Healthcare costs in patients using empagliflozin: an interim analysis from the EMPagliflozin compaRative effectiveness and SafEty (EMPRISE) Study. J Manag Care Spec Pharm. 2019;25(3-a Suppl):S39, (Abstract: E3).

27. Najafzadeh M, Pawar A, Déruaz Luyet A, et al. Reduced healthcare utilization in patients using empagliflozin: an interim analysis from the EMPagliflozin compaRative effectiveness and SafEty (EMPRISE) Study. J Manag Care Spec Pharm. 2019;25(3-a Suppl):S39 (Abstract:E2).

28. Liebl A, Khunti K, Orozco-Beltran D, Yale JF. Health economic evaluation of type 2 diabetes mellitus: a clinical practice focused review. Clin Med Insights Endocrinol Diabetes. 2015;8:13-9. 